\title{
Large-Scale Joint Rate and Power Allocation Algorithm Combined with Admission Control in Cognitive Radio Networks
}

\author{
Woo Jin Shin, Kyoung Youp Park, Dong In Kim, and Jang Woo Kwon
}

\begin{abstract}
In this paper, we investigate a dynamic spectrum sharing problem for the centralized uplink cognitive radio networks using orthogonal frequency division multiple access. We formulate a large-scale joint rate and power allocation as an optimization problem under quality of service constraint for secondary users and interference constraint for primary users. We also suggest admission control to find a feasible solution to the optimization problem. To implement the resource allocation on a large-scale, we introduce a notion of using the conservative factors $\alpha$ and $\beta$ depending on the outage and violation probabilities. Since estimating instantaneous channel gains is costly and requires high complexity, the proposed algorithm pursues a practical and implementationfriendly resource allocation. Simulation results demonstrate that the large-scale joint rate and power allocation incurs a slight loss in system throughput over the instantaneous one, but it achieves lower complexity with less sensitivity to variations in shadowing statistics.
\end{abstract}

Index Terms: Cognitive radio (CR), geometric programming, large-scale fading, orthogonal frequency division multiple access (OFDMA), outage probability, resource allocation, small-scale fading, violation probability.

\section{INTRODUCTION}

Orthogonal frequency division multiple access (OFDMA) is a promising modulation and access scheme for the proposed future wireless network standard like $4 \mathrm{G}$ cellular networks. For the network based on OFDMA, due to the large capacity that can be provided by OFDMA, it could be possible that the system is under-utilized and more profits can be obtained by exploiting cognitive radio (CR). In CR, unlicensed secondary users (SUs) can access the spectrum resources leased to the licensed primary users (PUs) with spectrum overlay and spectrum underlay so that limited spectrum resource can efficiently be utilized [1]. In spectrum overlay, SUs are allowed to access spectrum resources only when PUs do not use this resource. In spectrum underlay, unlicensed SUs can share the spectrum resource with licensed PUs under an interference limit (or spectral mask) defined as a maximum allowed interference at primary receiving points.

Manuscript received October 3, 2008.

The initial version of this paper will be presented in part at the IEEE VTC 2009 Spring, Barcelona, Spain.

This research was supported by the MKE, Korea, under the ITRC support program supervised by the IITA (IITA-2009-C1090-0902-0005).

W. J. Shin, K. Y. Park, and D. I. Kim are with the School of Information and Communication Engineering, Sungkyunkwan University, Suwon 440-746, Korea email: wjshin@skku.edu, \{kypark, dikim\}@ece.skku.ac.kr.

J. W. Kwon is with the Department of Computer Engineering, Tongmyoung University, Busan, Korea, email: jwkwon@tu.ac.kr.
To successfully deploy spectrum underlay based cognitive radio networks (CRNs), two apparently conflicting constraints must be satisfied simultaneously:

1) The quality of service (QoS) constraint (in terms of minimum required signal-to-interference-plus-noise ratio (SINR)) for SUs at each subcarrier.

2) Interference constraint for PUs to be protected from any harmful interference caused by SUs, that results from sharing the common spectrum resource.

Therefore, to meet these two constraints simultaneously, joint rate and power allocation algorithm combined with admission control is a prerequisite for the CRNs.

In literature, given information on all channel gains is available, resource allocation algorithms under QoS and interference constraints in code division multiple access (CDMA) system were proposed in [2] and [3]. However, from the practical point of view, it is difficult for low-end SUs to fast and accurately track the instantaneous channel gains. Moreover, the resource allocation adaptive to fast variations in the channel gains will increase the complexity associated with frequent measurements and updates. Especially, a threshold called the "spectral mask" should be predetermined to protect PUs from any harmful interference caused by SUs in CRNs. Consequently, in order to determine the spectral mask, an exact channel gain information is necessary so that the efficiency of the spectrum usage could be degraded because of the overhead caused by frequent exchanges of channel gain information across CRNs. More flexible approach that exploits the averaged channel gain was proposed in [4], where the outage and violation probabilities against smallscale fading for secondary and primary user links were introduced in single-carrier wideband code division multiple access (WCDMA) multi-cell model.

In this paper, we propose a large-scale joint rate and power allocation algorithm combined with admission control for uplink OFDMA based CRNs. We are concerned with the variations in large-scale fading and introduce the concept of outage and violation events against small-scale fast fading. This idea is similar to that given in [4]. However, this paper considers multi-cell CRNs with multi-carrier channel assignment, and admission control is also incorporated into the resource allocation algorithm to obtain a feasible solution to the optimization problem. In the process of solving the optimization problem, if any subcarrier assigned to a SU cannot be supported in terms of QoS, admission control is initiated to assure the QoS constraint on each SU's assigned subcarriers; otherwise we carry out the large-scale joint rate and power allocation without performing admission control. Our simulation results show that the 
proposed large-scale resource allocation algorithm can considerably reduce the complexity with a slight loss in throughput, and the two conservative $\alpha$ and $\beta$ factors have less sensitivity to variations in shadowing statistics which is desired for CR operation.

The rest of the paper is organized as follows. Section II describes the system model and defines mean channel gain. Largescale constraints are first defined and then the corresponding resource allocation problem is formulated in Section III. Section IV presents the geometric programming and its transformation method. The proposed large-scale joint resource allocation algorithm is described in Section V. Section VI verifies the performance of the proposed algorithm by simulations. Finally, concluding remarks are stated in Section VII.

\section{SYSTEM MODEL AND MEAN CHANNEL GAIN}

In this section, we first describe the system model, and then define mean channel gain which is averaged over small-scale fading.

\section{A. System Model}

We consider $M$ multi-cell OFDMA based CRNs where $L$ SUs and one base station (BS) exist in each cell and the total number of OFDM subcarriers is $K$. In each cell, subcarriers are assigned to SUs exclusively so that there is no intra-cell interference, whereas subcarriers are shared among different cells and this will cause inter-cell interference. In this paper, we assume that all channel information, such as path loss, shadowing and geo-location of PUs and SUs, is a priori known to each BS. Based on this information, each BS allocates resources to the SUs in its own cell.

For the uplink OFDMA, the SINR of the $i$ th SU in the $m$ th cell at subcarrier $k, \mu_{i(m), k}$ can be expressed as:

$$
\mu_{i(m), k}=\frac{g_{m, i(m), k}^{(s)} P_{i(m), k}}{\sum_{n=1, n \neq m}^{M} \sum_{j} g_{m, j(n), k}^{(s)} P_{j(n), k}+N_{i(m), k}}
$$

where $i(m)$ represents the $i$ th SU in the $m$ th cell, $g_{m, i(m), k}^{(s)}$ is the channel gain from the $i$ th SU to its corresponding BS in the $m$ th cell at subcarrier $k, g_{m, j(n), k}^{(s)}$ is the channel gain from the $j$ th SU in the $n$th cell to the BS in the $m$ th cell, $P_{i(m), k}$ is the transmitted power of the $i$ th SU in the $m$ th cell at subcarrier $k$, and $N_{i(m), k}$ is the $i$ th SU's noise power at subcarrier $k$. We assume that $N_{i(m), k}=N_{o} B_{k}$ for all SUs, where $N_{o}$ is one-sided noise power spectral density and $B_{k}$ is the $k$ th subcarrier bandwidth.

\section{B. Mean Channel Gain}

In general, it is difficult to estimate the instantaneous channel gains, and adjusting the power and rate allocation to the changes in small-scale fading could increase the system complexity and cost. For this reason, we assume that the mean channel gains (path loss and shadowing) averaged over small-scale fading (Rayleigh fading) are available for the resource allocation on a large-scale. The channel gain from the $i$ th SU to the corresponding $m$ th receiving point at subcarrier $k, g_{m, i(m), k}^{(s)}$ can be decomposed into

$$
g_{m, i(m), k}^{(s)}=\sigma_{m, i(m), k}^{(s)} \bar{g}_{m, i(m), k}^{(s)}
$$

where $\sigma_{m, i(m), k}^{(s)}$ is the small-scale fading with mean value normalized to one at subcarrier $k$, and $\bar{g}_{m, i(m), k}^{(s)}$ represents the mean channel gain, i.e., local average (with respect to smallscale fading) of $g_{m, i(m), k}^{(s)}$.

\section{LARGE-SCALE CONSTRAINT AND PROBLEM FORMULATION}

In this section, we define large-scale QoS and interference constraints, respectively. With defined large-scale constraints, we introduce the outage and violation probability constraints, respectively, and then formulate the large-scale optimization problem.

\section{A. Large-Scale QoS Constraint}

We can express an instantaneous QoS constraint for the $i$ th $\mathrm{SU}$ in the $m$ th cell at subcarrier $k$ as follows:

$$
\mu_{i(m), k} \geq \gamma_{i(m), k}
$$

where $\mu_{i(m), k}$ is an instantaneous SINR of the $i$ th SU in the $m$ th cell at subcarrier $k$, as given in (1) and $\gamma_{i(m), k}$ is the minimum required SINR for the $i$ th SU in the $m$ th cell at subcarrier $k$. By replacing the instantaneous channel gain in (1) with the mean channel gain defined in (2), we can express an averaged SINR of the $i$ th $\mathrm{SU}$ at subcarrier $k, \bar{\mu}_{i(m), k}$ as:

$$
\bar{\mu}_{i(m), k}=\frac{\bar{g}_{m, i(m), k}^{(s)} P_{i(m), k}}{\sum_{n=1, n \neq m}^{M} \sum_{j} \bar{g}_{m, j(n), k}^{(s)} P_{j(n), k}+N_{o} B_{k}} .
$$

With the averaged SINR, the large-scale QoS constraint can be formulated as

$$
\bar{\mu}_{i(m), k} \geq \alpha \gamma_{i(m), k}
$$

where $\alpha$ acts as a conservative factor that implies a kind of margin for the QoS constraint.

Then, we can define the outage event as that occurs when the instantaneous QoS constraint in (3) is not satisfied, given the large-scale QoS constraint in (5) is satisfied. Therefore, the outage probability constraint for large-scale QoS requirements on the secondary links is defined as:

$$
\operatorname{Pr}\left[\mu_{i(m), k}<\gamma_{i(m), k} \mid \bar{\mu}_{i(m), k} \geq \alpha \gamma_{i(m), k}\right] \leq \delta^{(s)}
$$

where some $\alpha>1$ and $\delta^{(s)}$ denotes the predetermined maximum outage probability allowed for SUs at each subcarrier.

\section{B. Large-Scale Interference Constraint}

Although the primary and secondary systems exploit different channel access schemes, since they share the common channel, total interference induced by SUs to primary receiving point $q$ 
can be obtained by a linear sum of all interferences at each subcarrier. The instantaneous sum interference induced by SUs to the primary receiving point $q, \eta_{q}$ and the instantaneous interference constraint can be expressed as follows:

$$
\begin{gathered}
\eta_{q}=\sum_{m=1}^{M} \sum_{i=1}^{L} \sum_{k \in \mathbf{S}_{\mathbf{i}(\mathbf{m})}} g_{q, i(m), k}^{(p)} P_{i(m), k} \\
\eta_{q} \leq T_{q}
\end{gathered}
$$

where $g_{q, i(m), k}^{(p)}$ is the channel gain from the $i$ th SU in the $m$ th cell to the primary receiving point $q$ at subcarrier $k, \mathbf{S}_{\mathbf{i}(\mathbf{m})}$ is the set of subcarriers assigned to the $i$ th $\mathrm{SU}$ and $T_{q}$ denotes the tolerable interference limit at primary receiving point $q, q=$ $1,2, \cdots, Q$.

Similar to the above large-scale QoS constraint case, if we replace the instantaneous channel gain in (7) by the mean channel gain, an averaged sum interference, $\bar{\eta}_{q}$ and the large-scale interference constraint can be formulated as:

$$
\begin{gathered}
\bar{\eta}_{q}=\sum_{m=1}^{M} \sum_{i=1}^{L} \sum_{k \in \mathbf{S}_{\mathbf{i}(\mathbf{m})}} \bar{g}_{q, i(m), k}^{(p)} P_{i(m), k} \\
\bar{\eta}_{q} \leq \beta T_{q}
\end{gathered}
$$

where $\beta$ acts as a conservative factor that implies a kind of margin for the interference constraint.

The violation probability constraint for large-scale interference limits on the primary receiving points can be defined as:

$$
\operatorname{Pr}\left[\eta_{q}>T_{q} \mid \bar{\eta}_{q} \leq \beta T_{q}\right] \leq \delta^{(p)}
$$

where some $\beta<1$ and $\delta^{(p)}$ denotes the predetermined maximum interference violation probability allowed for primary receiving points.

To ensure that the large-scale QoS and interference constraints defined in (5) and (10), respectively, are effective, the two conservative factors $\alpha>1$ and $\beta<1$ should be estimated to meet the constraints on outage and violation probabilities defined (6) and (11), respectively.

\section{Problem Formulation}

Our goal is to find an optimal resource allocation solution which maximizes the total sum rate of the system under the constraints (5), (6), (10), and (11). However, only after we obtain the solution satisfying the large-scale QoS and interference constraints, we can see whether the constraints on outage and violation probabilities are satisfied. For this reason, we divide a total resource allocation problem into two sub-problems such as large-scale optimization problem and outage and violation events check problem.

We first formulate the large-scale optimization problem under the maximum power constraint and large-scale constraints (i.e., QoS and interference constraints, (5) and (10), respectively). This large-scale optimization problem can be expressed as:

$$
\operatorname{maximize} \sum_{m=1}^{M} \sum_{i=1}^{L} \sum_{k \in \mathbf{S}_{\mathbf{i}(\mathbf{m})}} R_{i(m), k}
$$

subject to

$$
\begin{gathered}
\bar{\mu}_{i(m), k} \geq \alpha \gamma_{i(m), k} \\
\bar{\eta}_{q} \leq \beta T_{q} \\
\sum_{k \in \mathbf{S}_{\mathbf{i}(\mathbf{m})}} P_{i(m), k} \leq P_{i(m)}^{\max }
\end{gathered}
$$

where $R_{i(m), k}$ represents the $i$ th SU's data rate at subcarrier $k$, given by $R_{i(m), k}=B_{k} \log _{2}\left(1+\tau_{i(m)} \bar{\mu}_{i(m), k}\right), \tau_{i(m)}$ is an SNR gap of the $i$ th SU according to the modulation format and bit error rate (BER) requirement, $P_{i(m)}^{\max }$ is the maximum transmit power allowed to the $i$ th SU. In this paper, we assume that $B_{k}$ is the same for all subcarriers, i.e., $B_{k}=B$ and $\tau_{i(m)}$ is equal to 1 .

The above large-scale optimization problem is an intractable nonlinear optimization problem that may appear to be NP-hard problems [5], [6]. However, we will suggest the method to transform the proposed optimization problem into geometric programming (GP) which can be transformed into the convex optimization problem [5], [6]. In the next section, we discuss about the GP and then transform the optimization problem in (12) into GP.

\section{TRANSFORMATION OF THE OPTIMIZATION PROBLEM}

In this section, we first introduce the GP and then transform the proposed optimization problem into GP in standard form.

\section{A. Geometric Programming}

GP is a type of mathematical optimization problem which is nonlinear, non-convex. However, because an optimization problem formulated in GP format can be converted into a convex optimization problem, a local optimum could also be a global optimum and a global optimum can always be computed very efficiently [5], [6].

Let $\vec{x}=\left(x_{1}, x_{2}, \cdots, x_{n}\right)$ denote a vector with component $x_{i}$. A real valued function $f$ of $\vec{x}$ is defined as

$$
f(\vec{x})=c x_{1}^{d_{1}} x_{2}^{d_{2}} \cdots x_{n}^{d_{n}}
$$

where the multiplicative constant $c>0$ and the exponential components $d_{i} \in \mathbf{R}, i=1,2, \cdots, n$, is called a monomial function or monomial.

A function which is a sum of one or more monomials is called a posynomial function or posynomial and it can be expressed as

$$
f(\vec{x})=\sum_{k}^{K} c_{k} x_{1}^{d_{1(k)}} x_{2}^{d_{2(k)}} \cdots x_{n}^{d_{n(k)}}
$$

where $c_{k}>0$ and $d_{i(k)} \in \mathbf{R}$ for $i=1,2, \cdots, n$ and $k=$ $1,2, \cdots, K$.

GP in standard form is an optimization problem of the form which minimizes a posynomial objective function subject to posynomial upper bound inequality constraints (i.e., less than equal to one) and monomial equality constraints (i.e., equal to one):

$$
\operatorname{minimize} f_{0}(\vec{x})
$$


subject to

$$
\begin{gathered}
f_{i}(\vec{x}) \leq 1, i=1,2, \cdots, m, \\
g_{j}(\vec{x})=1, j=1,2, \cdots, p
\end{gathered}
$$

where $f_{i}, i=0,1, \cdots, m$ are posynomial functions, $g_{j}, j=$ $1,2, \cdots, p$ are monomial functions, and $\vec{x}$ is the optimization variable.

While GP in standard form is not a convex optimization problem, GP can be converted into the convex optimization problem with a logarithm change of the variables and multiplicative constants, and a logarithm transformation of the objective and constraint functions [6].

\section{B. Transformation Into Geometric Programming}

We need to transform the proposed optimization problem into GP in standard form to obtain a solution to the proposed optimization problem (12). To satisfy the condition on the GP in standard form, we make some manipulation of the objective function in (12). Maximizing the objective function expressed as the sum of $\log _{2}(1+\mathrm{SINR})$ in (12) is equivalent to minimizing the product of $1 /(1+\mathrm{SINR})$. Thus, we can convert the problem that maximizes the objective function into the equivalent one which minimizes the product of $1 /(1+\mathrm{SINR})$. However, the form of $1 /(1+\mathrm{SINR})$ is a ratio of two posynomials and minimizing a ratio of two posynomials is one of the truly nonconvex class of problems [7]. To solve this problem, two successive approximation methods, i.e., a logarithmic approximation method and a single condensation method, were introduced in [8], and hence we use these two successive approximation methods along with high SINR approximation as follows:

\section{1) High SINR approximation for GP}

If SINR is much greater than one, $\log _{2}(1+\mathrm{SINR})$ can be approximated to $\log _{2}(\mathrm{SINR})$. Therefore, $1 /(1+\mathrm{SINR})$ can approximately be converted into $1 / \mathrm{SINR}$ and the objective function is no longer a ratio of two posynomials. However, this approximation is reasonable only when the signal level is much higher than the interference level.

\section{2) Single condensation approximation method for GP [7]}

To solve the problem that the objective function is a ratio of two posynomials, we can employ the arithmetic-geometric mean inequality method which can transform the posynomial into the product of monomials. The arithmetic-geometric mean inequality indicates that $\sum_{i} a_{i} b_{i} \geq \prod_{i} b_{i}^{a_{i}}$, where $\vec{a} \succeq 0, \vec{b} \succ 0$, $\overrightarrow{1}^{T} \vec{a}=1$. Let $g_{i}=a_{i} b_{i}$ and then we can express this inequality as $\sum_{i} g_{i} \geq \prod_{i}\left(g_{i} / a_{i}\right)^{a_{i}}$. This inequality becomes tight with equality, if $a_{i}=g_{i} / \sum_{i} g_{i}$ for all $i$, which meet the condition that $\vec{a} \succeq 0$ and $\overrightarrow{1}^{T} \vec{a}=1$.

\section{3) Logarithmic approximation method for GP [9]}

A non-convex problem involving $\log _{2}(1+$ SINR $)$ can be approximated to $x \log _{2}(\mathrm{SINR})+y$ for some constants $x$ and $y$ satisfying the following conditions:

$$
x \log _{2}(z)+y \leq \log _{2}(1+z)
$$

$$
\begin{gathered}
x=\frac{z_{0}}{1+z_{0}} \\
y=\log _{2}\left(1+z_{0}\right)-\frac{z_{0}}{1+z_{0}} \log _{2}\left(z_{0}\right)
\end{gathered}
$$

where the inequality becomes tight with equality at a chosen value $z_{0}$, when the constants $x$ and $y$ are determined as specified above.

In the next subsection, we are going to select the most appropriate approximation method for our proposed optimization problem by comparing the results obtained from these three approximation methods.

\section{Two-User Two-Subcarrier Example}

Since the analytic comparison of the performance among these approximation methods seems to be intractable, in this paper we carry out a computer simulation under the same environment. The goal of the proposed optimization problem is to maximize the total sum rate of the system. Therefore, from the optimal point of view, it can be said that the method which yields the largest sum rate under the same large-scale QoS and interference constraints is the most suitable method for the proposed optimization problem. We compare the sum rates obtained from three different approximation methods:

1) High SINR approximation

2) single condensation approximation

3) logarithmic approximation

for the simple two-user two-subcarrier example.

In this example, we assume that each SU has the maximum transmit power $4 \mathrm{~W}$ and the same minimum required SINR, $3 \mathrm{~dB}$ at each subcarrier. The propagation model assumes the operation in a suburban environment and considers long-term fading (path loss and shadowing). Path loss exponent is set to 3.5 and shadowing for each SU is modeled as an independent lognormal random variable with standard deviation $6 \mathrm{~dB}$. The tolerable interference limit at the primary receiver is determined to yield the desired SINR of $23 \mathrm{~dB}$. To compare the sum rate achieved from each approximation method, we generate 100 different shadowing realizations under the same large-scale QoS and interference constraints with the fixed channel allocation and positions of two users.

From Fig. 1, we can see that logarithmic and single condensation approximation methods always perform better than high SINR approximation method in terms of the achieved sum rate. However, since the two logarithmic and single condensation methods result in almost similar outcome with a slight difference, the performance of these two approximation methods needs to be compared in detail, and we define a variable $\theta$ as

$$
\theta=S_{\log }-S_{s . c}
$$

where $S_{\log }$ indicates the total sum rate obtained from the logarithmic approximation method and $S_{s . c}$ represents the total sum rate achieved from the single condensation method.

In Fig. 2, we plot the instantaneous value of $\theta$ obtained from every trial as well as the average of $\theta$ over all trials. We can observe that not only the average of $\theta$ is greater than zero but 


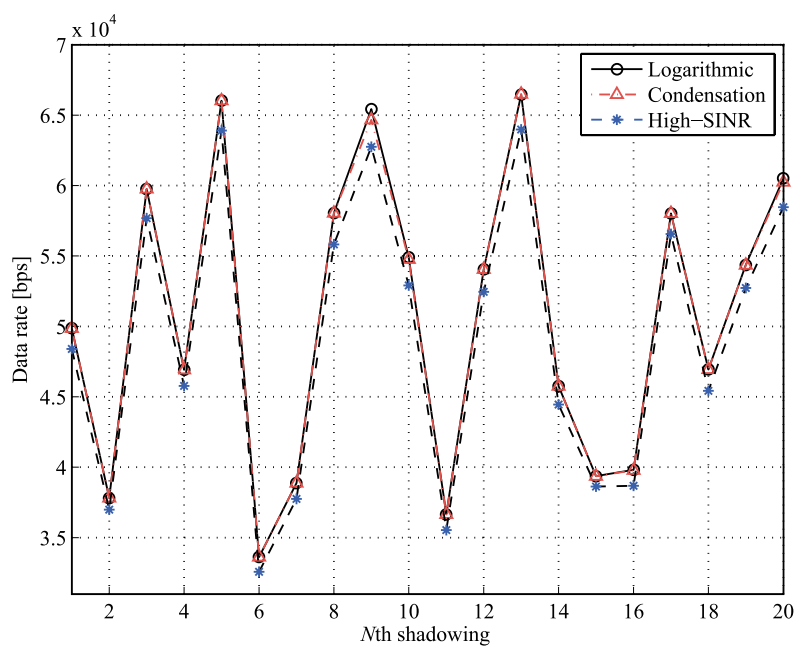

Fig. 1. Sum rates obtained from three different approximation methods.

also each value of $\theta$ is greater than zero in most simulation trials. Therefore, we could consider the logarithmic approximation method to be the most suitable for our proposed optimization problem based on the simulation results obtained from this two-user two-subcarrier example.

\section{Transformation with Logarithmic Approximation}

From the previous subsection, we verified that a logarithmic approximation method is the most suitable for our proposed optimization problem. Therefore, we employ the logarithmic approximation method to transform the objective function in our proposed optimization problem (12) into GP in standard form. From the transformation process, an optimal solution can be obtained by solving the following equivalent optimization problem which is GP in standard form:

$$
\operatorname{minimize} \prod_{m=1}^{M} \prod_{i=1}^{L} \prod_{k \in \mathbf{S}_{\mathbf{i}(\mathbf{m})}} \frac{1}{\bar{\mu}_{i(m), k}^{x_{i(m), k}} 2^{y_{i(m), k}}}
$$

subject to

$$
\begin{gathered}
\frac{\alpha \gamma_{i(m), k}}{\bar{g}_{m, i(m), k}^{(s)}} P_{i(m), k}^{-1} \sum_{n=1, n \neq m}^{M} \sum_{j} \bar{g}_{m, j(n), k}^{(s)} P_{j(n), k} \\
+\frac{\alpha \gamma_{i(m), k}}{\bar{g}_{m, i(m), k}^{(s)}} P_{i(m), k}^{-1} N_{o} B \leq 1, \\
\frac{\sum_{m=1}^{M} \sum_{i=1}^{L} \sum_{k \in \mathbf{S}_{\mathbf{i}(\mathbf{m})}} \bar{g}_{q, i(m), k}^{(p)} P_{i(m), k}}{\beta T_{q}} \leq 1, \\
\left(P_{i(m)}^{\max )^{-1}} \sum_{k \in \mathbf{S}_{\mathbf{i}(\mathbf{m})}} P_{i(m), k} \leq 1\right.
\end{gathered}
$$

where $x_{i(m), k}=\bar{\mu}_{i(m), k} /\left(1+\bar{\mu}_{i(m), k}\right)$ and $y_{i(m), k}=\log _{2}(1+$ $\left.\bar{\mu}_{i(m), k}\right)-\left(\bar{\mu}_{i(m), k} /\left(1+\bar{\mu}_{i(m), k}\right)\right) \log _{2}\left(\bar{\mu}_{i(m), k}\right)$. Values of $x_{i(m), k}$ and $y_{i(m), k}$ are determined by the iterative method as stated in [9].

A detailed procedure for transforming the optimization problem (12) into the equivalent one (18) can be found in the appendix.

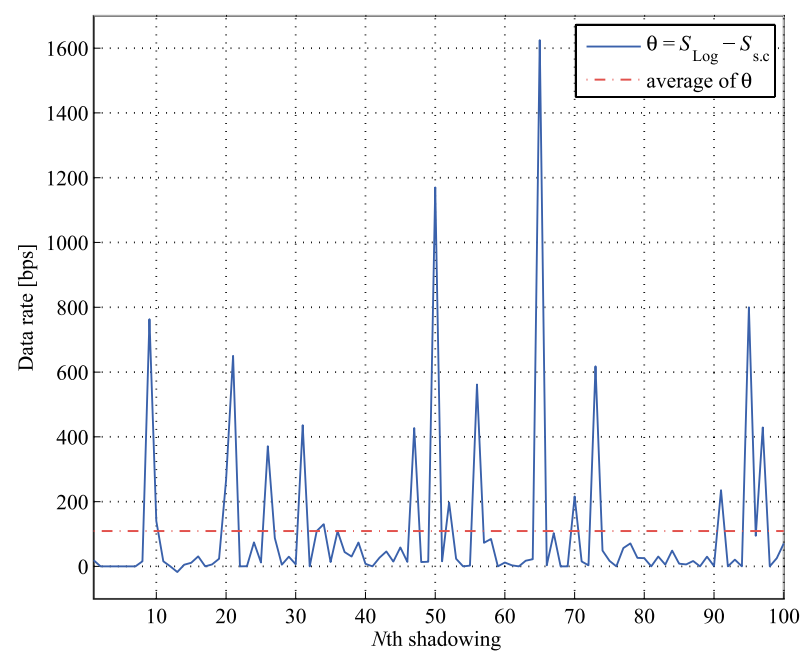

Fig. 2. Sum rate difference between logarithmic approximation and single condensation approximation methods.

Once the optimal solution is obtained from the optimization problem (18), we proceed to the second sub-problem, outage and violation events check problem. With the solution achieved from the optimization problem, we can evaluate the outage and violation probabilities to see if the obtained solution satisfies both outage and violation probability constraints, (6) and (11), respectively. If the obtained solution satisfies both outage and violation constraints, it is determined as a final optimal solution; otherwise we need to solve the optimization problem (18) again with newly updated $\alpha$ and $\beta$. The overall procedure will be described in the next section.

\section{LARGE-SCALE JOINT POWER AND RATE ALLOCATION WITH ADMISSION CONTROL}

In this section, we present the procedure of the proposed algorithm to find a solution to the optimization problem (18) under outage and violation probability constraints. Since the outage and violation probabilities depend on the solutions of the optimization problem, we propose the iterative algorithm to find out appropriate $\alpha$ and $\beta$ factors and the corresponding optimal solutions. The objective here is to maximize the total throughput while satisfying users' QoS requirements at each subcarrier. However, since higher transmit power of one user increases interference levels to other users, there may not be a feasible solution to meet QoS requirements for all users under the interference constraints. For this reason, we incorporate admission control into resource allocation algorithm such that QoS and interference constraints can be satisfied simultaneously.

The proposed large-scale resource allocation algorithm combined with admission control is designed as follows:

step 1: Initialize $\alpha=1$ and $\beta=1$.

step 2: Solve the large-scale optimization problem in (18) with the current $\alpha$ and $\beta$.

step 3: If a feasible solution satisfying both large-scale QoS and interference constraints is obtained, compute and store each user's SINR based on the solution obtained by current $\alpha$ and $\beta$, and then go to step 4; otherwise go to step 6 . 
step 4: Compute the outage and violation probabilities and check whether they satisfy both outage and violation probabilities constraints in (6) and (11), respectively. If the feasible solution obtained from step 3 satisfies both outage and violation constraints, finish and allocate obtained resources to the users; otherwise go to step 5.

step 5: Update the conservative factors, $\alpha$ and $\beta$ as follows:

if outage constraint in (6) is violated, $\alpha=\alpha+\Delta \alpha$

if violation constraint in (11) is violated, $\beta=\beta-\Delta \beta$

where $\Delta \alpha$ and $\Delta \beta$ are predetermined small adjustment values.

After updating $\alpha$ and $\beta$, go back to step 2 with the updated $\alpha$ and $\beta$.

step 6: (Admission control) If a currently used $\alpha$ is 1 , solve the optimization problem in step 2 again without QoS constraint (i.e. setting $\alpha$ to be zero) and calculate SINR to set a criterion for the admission control; otherwise use the SINR measured in step 3 as a criterion for the admission control.

step 7 : (Admission control) Perform the admission control to prevent the worst user producing the lowest SINR of all users and all subcarriers from using the corresponding subcarrier. After step 7, return to step 2 .

The process of estimating appropriate $\alpha$ and $\beta$ satisfying outage and violation probability constraints (i.e., step 4 and 5) does not need to be done again as long as the given network condition and channel allocation do not change. Therefore, the proposed algorithm can be implemented in two modes such as tuning mode and blind mode. In a given network and channel allocation, the proposed algorithm will do all steps (i.e., from step 1 to 7) during the tuning mode. Through tuning mode, we can estimate the proper $\alpha$ and $\beta$ and the corresponding solutions to the optimization problem. For the blind mode, since the $\alpha$ and $\beta$ obtained from tuning mode can continuously be used (while the given network condition and channel allocation do not change), we are required to implement only several steps (i.e., step 2, 3,6 , and 7). Note that if outage and/or violation occur during the blind mode, adaptive modulation and coding (AMC) may be implemented to adjust the data rate for a given QoS at each subcarrier.

\section{SIMULATION RESULTS}

We evaluate the performance of the proposed algorithm in IEEE 802.22 Wireless Regional Area Network (WRAN) which is the first cognitive radios based wireless standard [10], [11]. We consider the co-existence scenario where one primary network and two secondary network coexist. To obtain a protect contour and keep-out region defined in [11], we assume that the distance from a primary receiver to the BS in each secondary network is $26 \mathrm{~km}$ and two cells are apart from each other with the distance of $4 \mathrm{~km}$. In each cell, one BS is located at the center of the cell and three SUs are randomly generated at the edge of the cell to make the sum interference to primary receiver severe. The propagation model assumes the operation in a suburban environment and takes into consideration path loss and shadowing. Path loss exponent is set to be 3.5 and shadowing for each SU is modeled as an independent log-normal random variable with standard deviation $6 \mathrm{~dB}$ and $8 \mathrm{~dB}$. The ten-path Rayleigh fading

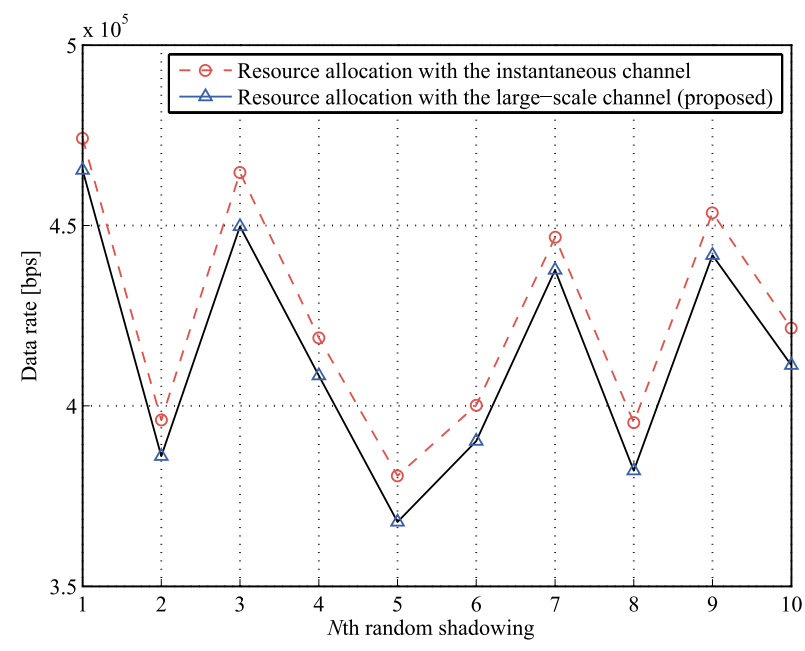

Fig. 3. Sum rate comparison of the proposed algorithm and instantaneous resource allocation method.

model is considered to simulate the frequency selective fading channel. We consider OFDMA system with 12 subcarriers in total and 4 subcarriers are randomly allocated to each SU in each cell. In each cell, subcarriers are assigned to SUs exclusively so that there is no intra-cell interference. However, subcarriers are shared among different cells and this will cause inter-cell interference. The overall bandwidth is $0.36 \mathrm{MHz}$ (i.e., $3 \mathrm{KHz}$ per sub-channel). We assume that all SUs have the same total transmit power, $4 \mathrm{~W}$ and the same minimum required SINR, $3 \mathrm{~dB}$ at every subcarrier. Maximum outage and interference violation probability is set to be $5 \%$ and $0.5 \%$, respectively. The adjustment values for conservative factors are empirically chosen to be $\Delta \alpha=1$ and $\Delta \beta=0.05$. The tolerable interference limit at the primary receiver is determined to yield the desired SINR of $23 \mathrm{~dB}$. To check whether the solutions obtained from the optimization problem in (18) satisfy outage and violation probabilities, we generate 10,000 different small-scale fading events.

\section{A. Throughput Performance}

To investigate the throughput performance of the proposed algorithm, we compare the sum rate obtained from the proposed algorithm to the one obtained from allocating resources according to the instantaneous channel gains. We assume that the frame length is on the order of 10 to 20 msec while the channel coherence time is typically on the order of $2.5 \mathrm{msec}$ [12], in which case small-scale fading changes 4 to 8 times every frame transmission. Typically, large-scale fading changes once every tens of frames, depending on the propagation environments, and hence we assume that small-scale fading approximately changes 100 times per large-scale fading. The sum rate of the instantaneous resource allocation is obtained by taking the average of data rates achieved from each trial.

In Fig. 3, we can see that the sum rate of the proposed algorithm incurs about $2.64 \%$ of loss when it compares to the sum rate of the instantaneous resource allocation. Since $5 \%$ outage probability is assumed in the proposed algorithm, it will incur additional loss in the outage compared to the instantaneous resource allocation. However, the proposed algorithm 


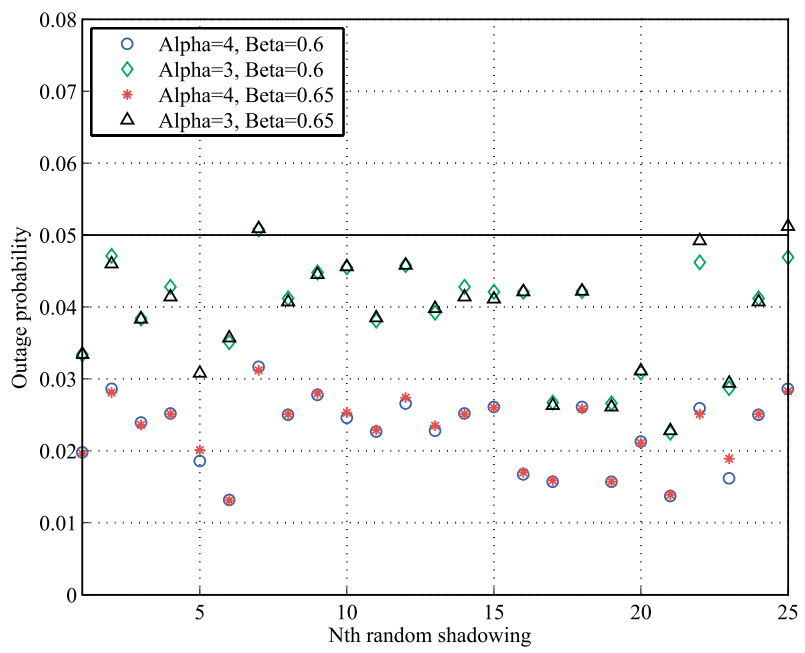

Fig. 4. Outage probability for shadowing with standard deviation $6 \mathrm{~dB}$.

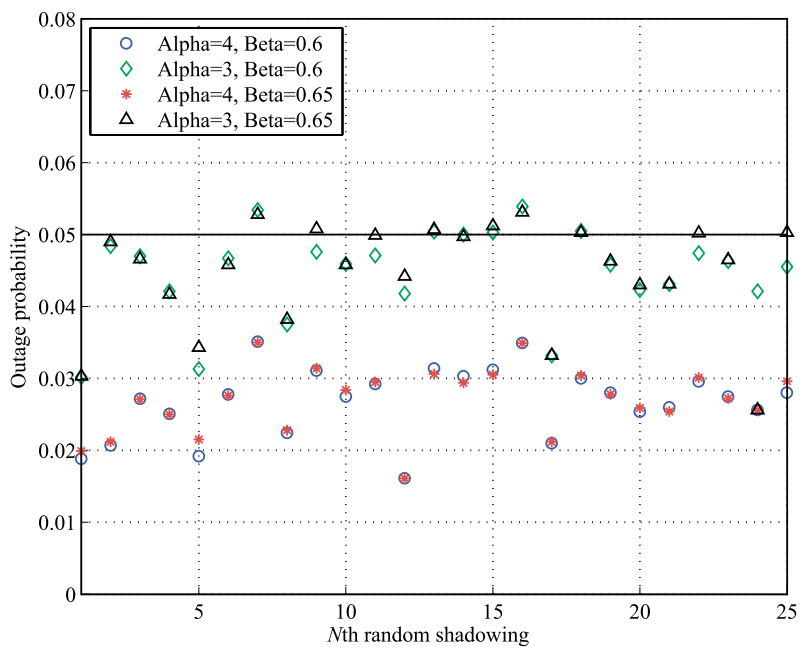

Fig. 5. Outage probability for shadowing with standard deviation $8 \mathrm{~dB}$.

still achieves 1/100 times less frequent power adaptation with a slight loss in sum rate. Also, the simulation results show that the proposed algorithm is not sensitive to the ratio factor between large-scale and small-scale fading as long as it ranges from 10 to 1000 .

\section{B. Sensitivity of $\alpha$ and $\beta$ to the Variations in Shadowing}

To investigate whether the achieved $\alpha$ and $\beta$ are sensitive to variations in shadowing, we simulate the outage and violation probabilities with the different shadowing whose standard deviation is $6 \mathrm{~dB}$ and $8 \mathrm{~dB}$, respectively. In Figs. 4-7, we present the computed outage probability and violation probability with standard deviation $6 \mathrm{~dB}$ and $8 \mathrm{~dB}$, respectively. From the simulation results, when estimated $\alpha=4$ and $\beta=0.6$, we can observe that every outcome of all trials satisfies both maximum outage and violation probabilities in both cases where the standard deviation of shadowing is $6 \mathrm{~dB}$ and $8 \mathrm{~dB}$, respectively. Therefore, by estimating proper $\alpha$ and $\beta$ during the training mode, the estimated $\alpha$ and $\beta$ can reliably be used during the blind mode even with slight variations in shadowing, so that we do not need to

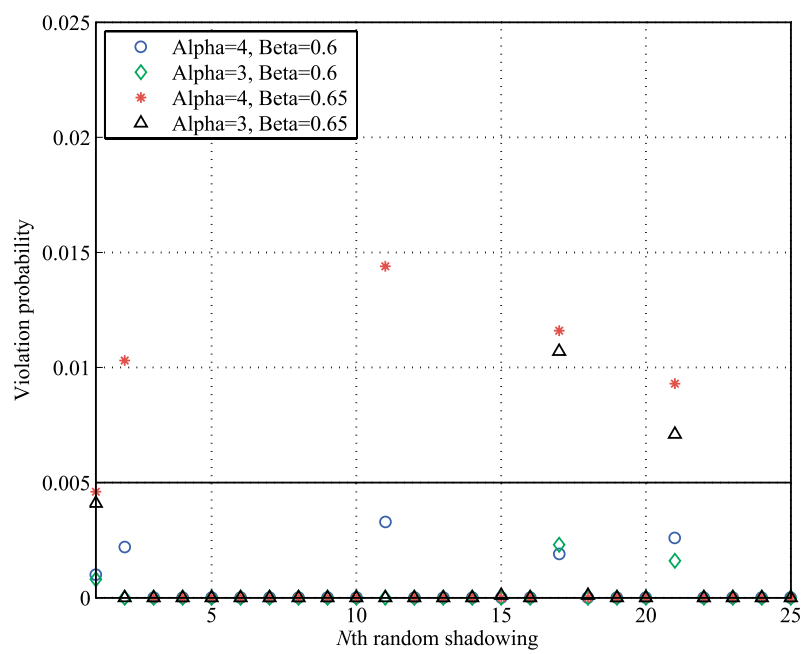

Fig. 6. Violation probability for shadowing with standard deviation $6 \mathrm{~dB}$.

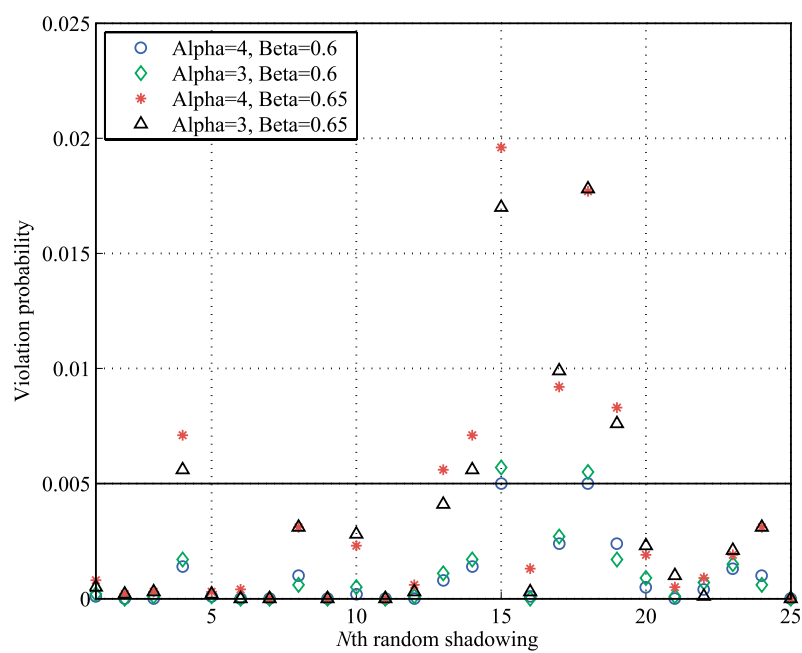

Fig. 7. Violation probability for shadowing with standard deviation $8 \mathrm{~dB}$.

estimate $\alpha$ and $\beta$ frequently, resulting in reduced overhead.

\section{CONCLUSION}

We have proposed a framework for dynamic spectrum sharing between primary and secondary networks. To overcome the complexity problem caused by tracking the channel gains instantaneously, the proposed algorithm allocates resources to users on a large-scale while satisfying both large-scale QoS and interference constraints under the constraints on the outage and violation probabilities. Introducing two conservative factors $\alpha$ and $\beta$ makes it possible for the system to implement more flexible power allocation over conventional one, leading to a practical and implementation-friendly resource allocation for CRNs. As the large-scale fading changes less, the effectiveness of the proposed algorithm becomes better. Therefore, the proposed algorithm could be suitable for the IEEE 802.22 WRAN where customer premises equipments (which referred to as users in this paper) are considered to be fixed terminals. Further extension of the proposed algorithm to ad-hoc based CRNs and considering 
user-based fairness in the optimization problem are worth pursing.

\section{APPENDIX}

By logarithmic approximation (16), the objective function in (12) can be expressed as:

$$
\begin{aligned}
& \sum_{m=1}^{M} \sum_{i=1}^{L} \sum_{k \in \mathbf{S}_{\mathbf{i}(\mathbf{m})}} R_{i(m), k}=\sum_{m=1}^{M} \sum_{i=1}^{L} \sum_{k \in \mathbf{S}_{\mathbf{i}(\mathbf{m})}} B_{k} \log _{2}\left(1+\bar{\mu}_{i(m), k}\right) \\
& =\sum_{m=1}^{M} \sum_{i=1}^{L} \sum_{k \in \mathbf{S}_{\mathbf{i}(\mathbf{m})}} B_{k}\left(x_{i(m), k} \log _{2}\left(\bar{\mu}_{i(m), k}\right)+y_{i(m), k}\right) \\
& =\sum_{m=1}^{M} \sum_{i=1}^{L} \sum_{k \in \mathbf{S}_{\mathbf{i}(\mathbf{m})}} B_{k}\left(\log _{2}\left(\bar{\mu}_{i(m), k}\right)^{x_{i(m), k}}+\log _{2}\left(2^{y_{i(m), k}}\right)\right) \\
& =\sum_{m=1}^{M} \sum_{i=1}^{L} \sum_{k \in \mathbf{S}_{\mathbf{i}(\mathbf{m})}} B_{k} \log _{2}\left(\left(\bar{\mu}_{i(m), k}\right)^{x_{i(m), k}} 2^{\left.y_{i(m), k}\right)}\right.
\end{aligned}
$$

where $x_{i(m), k}=\bar{\mu}_{i(m), k} /\left(1+\bar{\mu}_{i(m), k}\right)$ and $y_{i(m), k}=\log _{2}(1+$ $\left.\bar{\mu}_{i(m), k}\right)-\left(\bar{\mu}_{i(m), k} /\left(1+\bar{\mu}_{i(m), k}\right)\right) \log _{2}\left(\bar{\mu}_{i(m), k}\right)$.

We assume that the bandwidth of all subcarriers is the same, i.e., a constant value $B_{k}=B$, and $\log _{2}(x)$ is an increasing function with value of $x>1$. Therefore, with the approximated objective function in (19), the optimization problem (12) can be formulated as:

$$
\operatorname{maximize} \prod_{m=1}^{M} \prod_{i=1}^{L} \prod_{k \in \mathbf{S}_{\mathbf{i}(\mathbf{m})}}\left(\left(\bar{\mu}_{i(m), k}\right)^{x_{i(m), k}} 2^{y_{i(m), k}}\right)
$$

subject to

$$
\begin{gathered}
\frac{\bar{g}_{m, i(m), k}^{(s)} P_{i(m), k}}{\sum_{n=1, n \neq m}^{M} \sum_{j} \bar{g}_{m, j(n), k}^{(s)} P_{j(n), k}+N_{o} B} \geq \alpha \gamma_{i(m), k}, \\
\sum_{m=1}^{M} \sum_{i=1}^{L} \sum_{k \in \mathbf{S}_{\mathbf{i}(\mathbf{m})}} \bar{g}_{q, i(m), k}^{(p)} P_{i(m), k} \leq \beta T_{q}, \\
\sum_{k \in \mathbf{S}_{\mathbf{i}(\mathbf{m})}} P_{i(m), k} \leq P_{i(m)}^{\max } .
\end{gathered}
$$

To satisfy the condition on the objective function of GP in standard form, the optimization problem stated (20) can be transformed into the equivalent problem which is GP in standard form:

$$
\operatorname{minimize} \prod_{m=1}^{M} \prod_{i=1}^{L} \prod_{k \in \mathbf{S}_{\mathbf{i}(\mathbf{m})}} \frac{1}{\left(\bar{\mu}_{i(m), k}\right)^{x_{i(m), k}} 2^{y_{i(m), k}}}
$$

subject to

$$
\begin{gathered}
\frac{\alpha \gamma_{i(m), k}}{\bar{g}_{m, i(m), k}^{(s)}} P_{i(m), k}^{-1} \sum_{n=1, n \neq m}^{M} \sum_{j} \bar{g}_{m, j(n), k}^{(s)} P_{j(n), k} \\
+\frac{\alpha \gamma_{i(m), k}}{\bar{g}_{m, i(m), k}^{(s)}} P_{i(m), k}^{-1} N_{o} B \leq 1,
\end{gathered}
$$

$$
\begin{gathered}
\frac{\sum_{m=1}^{M} \sum_{i=1}^{L} \sum_{k \in \mathbf{S}_{\mathbf{i}(\mathbf{m})}} \bar{g}_{q, i(m), k}^{(p)} P_{i(m), k}}{\beta T_{q}} \leq 1, \\
\left(P_{i(m)}^{\max }\right)^{-1} \sum_{k \in \mathbf{S}_{\mathbf{i}(\mathbf{m})}} P_{i(m), k} \leq 1 .
\end{gathered}
$$

\section{REFERENCES}

[1] Q. Zhao and B. M. Sadler, "A survey of dynamic spectrum access: Signal processing, networking, and regulatory policy," IEEE Signal Process. Mag., pp. 79-89, May 2007.

[2] D. I. Kim, E. Hossain, and V. K. Bhargava, "Downlink joint rate and power allocation in cellular multi-rate WCDMA systems," IEEE Trans. Wireless Commun., vol. 2, pp. 69-80, Jan. 2003.

[3] L. Le and E. Hossain, "Resource allocation for spectrum underlay in cognitive radio networks," IEEE Trans. Wireless Commun., vol. 7, pp. 53065315, Dec. 2008.

[4] D. I. Kim, L. Le, and E. Hossain, "Joint rate and power allocation for cognitive radios in dynamic spectrum access environment," IEEE Trans. Wireless Commun., vol. 7, pp. 5517-5527, Dec. 2008.

[5] S. Boyd and L. Vandenberge, Convex Optimization, Cambridge University Press, 2004.

[6] S. Boyd, S. J. Kim, L. Vandenberghe, and A. Hassibi, A Tutorial on Geometric Programming. [Online]. Available: www.stanford.edu/ boyd/research.html

[7] M. Chiang. C. W. Tan. "Power control by geometric programming," IEEE Trans. Wireless Commun., vol. 6, pp. 2640-2651, July 2007.

[8] Z.-Q. Luo and W. Yu, "An introduction to convex optimization for communications and signal processing," IEEE J. Sel. Areas Commun., vol. 24, pp. 1426-1438, Aug. 2006.

[9] J. Papandriopoulos and J. Evans, "Low-complexity distributed algorithms for spectrum balancing in multi-user DSL networks," in Proc. IEEE ICC, vol. 7, June 2006, pp. 3270-3275.

[10] C. Cordeiro, K. Challapali, D. Birru, and Sai Shankar, "IEEE 802.22: The first worldwide wireless standard based on cognitive radios," in Proc.IEEE DySPAN, Nov. 2005, pp. 328-337.

[11] C. Cordeiro et al., "IEEE 802.22: An introduction to the first wireless standard based on cognitive radios," J. Commun., vol. 1, pp. 38-47, Apr. 2006.

[12] D. Tse and P. Viswanath, Fundamentals of Wirelesss Communications, Cambridge University Press, 2005

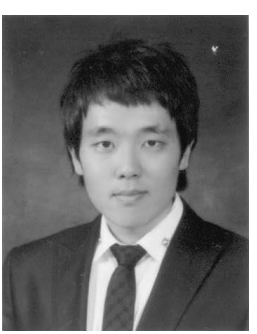

Woo Jin Shin received the B.S. degree in Information and Communication Engineering from Sungkyunkwan University, Suwon, Korea in 2008. He is currently pursuing his M.S. degree in the Department of Information and Communication Engineering at Sungkyunkwan University. His research interests include optimization, resource allocation, multi-cell cognitive radio networks, and cooperative communications.

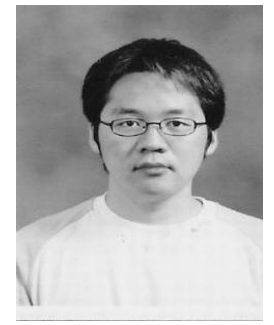

Kyoung Youp Park received the B.S. degree in Electronic and Electrical Engineering from the Hanyang University, Ansan, Korea in 2008. He is currently a M.S. candidate in the Department of Information and Communication Engineering at Sungkyunkwan University. His research interests include OFDM systems, radio resource allocation, cross-layer design of cognitive radio systems, and optimization. 


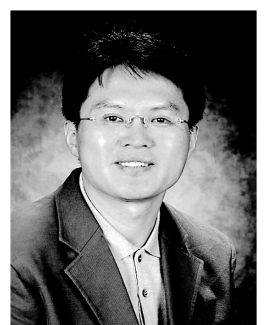

Dong In Kim received the B.S. and M.S. degrees in Electronics Engineering from Seoul National University, Seoul, Korea, in 1980 and 1984, respectively, and the M.S. and Ph.D. degrees in Electrical Engineering from University of Southern California (USC), Los Angeles, in 1987 and 1990, respectively. From 1984 to 1985 , he was a Researcher with Korea Telecom Research Center, Seoul. From 1986 to 1988, he was a Korean Government Graduate Fellow in the Department of Electrical Engineering, USC.

From 1991 to 2002, he was with the University of Seoul, Seoul, leading the Wireless Communications Research Group. From 2002 to 2007, he was a tenured Full Professor in the School of Engineering Science, Simon Fraser University, Burnaby, BC, Canada. From 1999 to 2000, he was a Visiting Professor at the University of Victoria, Victoria, BC. Since 2007, he has been with Sungkyunkwan University (SKKU), Suwon, Korea, where he is a Professor and SKKU Fellow in the School of Information and Communication Engineering. Since 1988, he is engaged in the research activities in the areas of wideband wireless transmission and access. His current research interests include cooperative relaying and base station (BS) cooperation, multiuser cognitive radio networks, advanced transceiver design, and cross-layer design.

He was an Editor for the IEEE Journal on Selected Areas in Communications: Wireless Communications Series and also a Division Editor for the Journal of Communications and Networks. He is currently an Editor for Spread Spectrum Transmission and Access for the IEEE Transactions on Communications and an Area Editor for Transmission Technology III for the IEEE Transactions on Wireless Communications. He also serves as Co-Editor-in-Chief for the Journal of Communications and Networks.

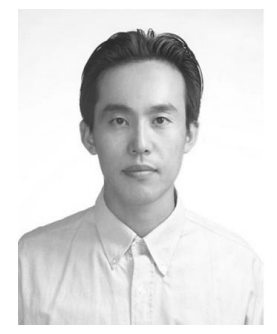

Jang Woo Kwon received the B.S degree in electronic Eng. from INHA University in 1990, the M.E. and $\mathrm{Ph} . \mathrm{D}$. degree in electronic engineering from INHA University in 1992 and 1996, respectively.

In 1992 he was a visiting Researcher at Department of Biomedical Engineering of Tokyo University. From 1996 to 1998 he was a Judge of Korea Industrial Property Office (KIPO). In 1998 he became a faculty member of Tongmyoung University. He has been a Dean of Research Institute for Information Eng. Tech. at Tongmyoung University since 2002. From 2003 to 2006, he is a Dean of Internet Broadcasting Station and Information Processing Center of T.I.T. From 2004 to present he is an Associate Professor of Department of Computer Eng. at Tongmyoung University. From 2006 he has been Director of Human Resource Development Division of Institute for Information Technology Advancement. Research topics include intelligent system using artificial neural network, web application, prosthesis arm control, movement trajectory formation and robot manipulators control based on bio signal analysis.

$\mathrm{He}$ is an Editor-in-Chief of the Institute Korea Information Processing Society (KIPS), Korean Institute of Information Scientists and Engineers (KIISE). $\mathrm{He}$ is also invited Judge for KIPO and consultant member for Informational Apparatus Standard. For the last 15 years he has been working in bio signal analysis and its recognition using artificial intelligence. Currently, he has interested in $\mathrm{u}-$ health care and HCI using bio signal. He has published about 100 papers. He was awarded an outstanding paper award from the Korean Sensors Society in 1995. He was given a citation from the Minister of Ministry of Maritime and Affairs and Fisheries (MOMAF) for his excellent service to MOMAF in April 2001. He was also given a citation from the President of a T.I.T. for his effort to T.I.T in March 2001. 